\title{
Microarray and bioinformatics analyses of gene expression profiles in BALB/c murine macrophage polarization
}

\author{
LI JIANG $^{1 *}$, XUEQIN LI ${ }^{2 *}$, YINGYING ZHANG ${ }^{3 *}$, MENGYING ZHANG $^{2}$, ZONGSHENG TANG $^{2}$ and $\mathrm{KUN}^{2} \mathrm{LV}^{2}$ \\ ${ }^{1}$ Department of Pharmacy, Institute of Dermatology, Chinese Academy of Medical Sciences, Nanjing, Jiangsu 210001; \\ ${ }^{2}$ Central Laboratory of Yijishan Hospital; ${ }^{3}$ Laboratory Medicine of Yijishan Hospital, \\ Wannan Medical College, Wuhu, Anhui 241001, P.R. China
}

Received February 23, 2016; Accepted February 9, 2017

DOI: $10.3892 / \mathrm{mmr} .2017 .7511$

\begin{abstract}
Macrophages possess the hallmark feature of plasticity, allowing them to undergo a dynamic transition between M1 and M2 polarized phenotypes. The aim of the present study was to screen for differentially-expressed genes (DEGs) that were associated with BALB/c murine macrophage polarization. The transcription profiles of three M1 and three M2 samples were obtained using microarray analysis. Based on the threshold of fold-change $>2.0$ and P-value $<0.05$, a total of 1,253 DEGs were identified, of which 696 were upregulated and 557 downregulated in M1 macrophages compared with M2 macrophages. Gene Ontology (GO) function and Kyoto Encyclopedia of Genes and Genomes (KEGG) pathway enrichment analyses were performed. A gene-gene interaction network of the DEGs was constructed using the Search Tool for the Retrieval of Interacting Genes database. GO annotation identified three categories: Cellular component, molecular function and biological process, with 34 and 40 enrichment terms consisting of upregulated and downregulated DEGs, respectively. GO enrichment analysis of DEGs was primarily associated with protein binding, response to stimulus, cell differentiation, and regulation of biological process. KEGG enrichment identified 15 and four pathways involving upregulated and downregulated DEGs, respectively. Signaling pathway analysis revealed that these DEGs were mainly involved in apoptosis, hypoxia-inducible factor (HIF) 1a pathway, innate immune system, tumor necrosis factor (TNF) signaling pathway, cytokine-cytokine receptor interaction, and other signal transduction pathways. Interaction network analysis indicated that genes including TNF, interleukin
\end{abstract}

Correspondence to: Dr Kun Lv, Central Laboratory of Yijishan Hospital, Wannan Medical College, 2 Western Zheshan Road, Wuhu, Anhui 241001, P.R. China

E-mail: 1vkun315@126.com

${ }^{*}$ Contributed equally

Key words: macrophage polarization, expression profile, microarray, bioinformatics, interaction
(IL)-6, IL-1 $\beta$, suppressor of cytokine signaling 3, nitric oxide synthase 2, HIF1a may serve key roles in macrophage polarization. The present study provided new insights into the role of genes in macrophage differentiation and polarization.

\section{Introduction}

Macrophages are derived from hematopoietic stem cells, in particular, from bone marrow myeloid progenitor cells. Beyond the classical functions of pathogen elimination, tissue development and wound repair, macrophages are well-recognized key regulators of both innate and adaptive immunity, as well as important mediators of systemic metabolism, angiogenesis, apoptosis, malignancy and reproduction (1-3). Macrophages display a high degree of plasticity, with the ability to generate different functional phenotypes (namely M1 and M2) in response to microenvironmental cues $(4,5)$. Cytokines and microbial products have been implicated in the reprogramming of M1 and M2 macrophages: Lipopolysaccharide (LPS) plus interferon (IFN)- $\gamma$ induce M1 macrophage activation, while stimulation of macrophages with interleukin (IL)-4 or IL-13 induces M2 macrophage activation (6,7). M1 macrophages secrete tumor necrosis factor (TNF)- $\alpha$, IL12 and IL-23, as well as large amounts of nitric oxide by expressing inducible nitric oxide synthase, which are essential for clearing bacterial, viral and fungal infections and in mediating resistance against tumors (8). M2 macrophages are characterized by upregulation of arginase (Arg)1, chitinase 3-like 3 (CHI3L3), resistin-like $\alpha$ (Retnla), mannose receptor C (Mrc)-1 (also known as CD206) and chemokines such as $\mathrm{C}-\mathrm{C}$ motif chemokine ligand (CCL)17 and CCL24. They are important in the host response to parasite infection, tissue remodeling, angiogenesis and tumor progression (9-12).

Macrophage polarization has been the focus of previous studies, particularly with regards to transcriptional regulation. Transcriptional factors, such as nuclear factor- $\kappa \mathrm{B}$, Jun proto-oncogene AP-1 transcription factor subunit, signal transducer and activator of transcription (STAT) 1, interferon regulatory factor (IRF)3, IRF5, IRF8, hypoxia-inducible factor (HIF) 1a, Kruppel-like factor (KLF) 2 and AKT serine/threonine kinase 1 (AKT1) participate in toll-like receptor (TLR)-induced M1 activation (8,13-17). In contrast, STAT6, IRF4, HIF2 $\alpha$, peroxisome proliferator-activated 
receptor (PPAR)- $\gamma$, CCAAT/enhancer-binding protein $\beta$, glucocorticoid receptors, AKT2, and KLF4 are involved in the polarization of macrophages to the M2 phenotype $(8,13-17)$. microRNA (miRs), such as miR-27b and miR-155, are involved in M1 polarization, whereas miR-9, miR-21, miR-125b, miR-146a, miR-223, Let-7i, Let-7c and Let-7e are involved in M2 macrophage polarization $(1,2,6,18)$. In addition, enzymes involved in epigenetic regulation, such as Jumonji domain-containing 3 (JMJD3) and histone deacetylase 3, are important in M2 macrophage polarization (19-21). Furthermore, the importance of suppressor of cytokine signaling (SOCS) 2 and SOCS3 proteins in M1 and M2 macrophage polarization has been recently demonstrated (22).

Microarray and bioinformatics analyses are effective ways of identifying genes and interactions between genes $(23,24)$. The present study utilized microarray and bioinformatics approaches to identify differentially-expressed genes (DEGs) and to analyze the gene expression features of ex vivo polarized M1 and M2 macrophages. Several molecular markers of each macrophage polarization phenotype were observed, thereby providing a theoretical basis for further experimental studies.

\section{Materials and methods}

Mice. A total of $20 \mathrm{BALB} / \mathrm{c}$ male mice (6-8 weeks old, 25-30 g) were obtained from the Experimental Animal Center of Qinglongshan (Nanjing, China), and were housed in pathogen-free mouse colonies with a 12-h light, 12-h dark cycle. Mice received standard chow diet, with free access to drinking water between 25 and $26^{\circ} \mathrm{C}$. Relative humidity was maintained between 60 and $70 \%$, and padding was changed twice/week. All animal experiments were performed according to the guidelines for the Care and Use of Laboratory Animals (Ministry of Health, China, 1998). All experimental protocols were approved by the Animal Ethics Committee of Yijishan Hospital (Wuhu, Anhui, China).

Cell culture and stimulation. Bone marrow-derived macrophages (BMDMs) were isolated from BALB/c mice by flushing the femurs with Dulbecco's modified Eagle's medium (DMEM; HyClone; GE Healthcare, Chicago, IL, USA) according to our previous studies $(6,25)$. Ethical approval was provided by the Animal Ethical Committee of Yijishan Hospital. Macrophages plated on six-well plates $\left(1 \times 10^{6}\right.$ cells/well $)$ were maintained in DMEM supplemented with $20 \%$ fetal bovine serum (FBS; Gibco; Thermo Fisher Scientific, Inc., Waltham, MA, USA) and $20 \% \mathrm{~L} 929$ supernatant at $37^{\circ} \mathrm{C}$ and $5 \% \mathrm{CO}_{2}(26)$. Following 7 days in culture, the medium was removed, and the cells were cultured in RPMI-1640 (HyClone; GE Healthcare) supplemented with 10\% FBS for an additional $24 \mathrm{~h}$. Macrophages were then stimulated for $48 \mathrm{~h}$ in DMEM/10\% FBS containing either $100 \mathrm{ng} / \mathrm{ml}$ LPS and $20 \mathrm{ng} / \mathrm{ml}$ IFN- $\gamma$ (for M1 polarization) or $20 \mathrm{ng} / \mathrm{ml} \mathrm{IL-4} \mathrm{(for} \mathrm{M2} \mathrm{polarization),} \mathrm{as} \mathrm{described}$ previously $(6,25)$.

RNA extraction and purification. BMDMs were collected following $48 \mathrm{~h}$ culture with polarization stimuli, and total RNA was extracted using TRIzol (Invitrogen; Thermo Fisher Scientific, Inc.), according to the manufacturer's instructions. RNA quantity and quality were measured using a
NanoDrop 2000 (Thermo Fisher Scientific, Inc.), and RNA integrity was assessed using an Agilent Bioanalyzer 2100 (Agilent Technologies, Inc., Santa Clara, CA, USA) and denaturing agarose gel electrophoresis. Total RNA was further purified using an RNeasy Mini kit and RNase-Free DNase set (both from Qiagen GmbH, Hilden, Germany).

Microarray analysis. Total RNA from each sample was amplified and labeled by using a Low Input Quick Amp WT Labeling kit (Agilent Technologies), following the manufacturer's instructions. Labeled cRNA was purified using an RNeasy Mini kit (Qiagen $\mathrm{GmbH}$ ). The concentration and specific activity of the labeled cRNAs (pmol Cy3/ $/ \mu \mathrm{g}$ cRNA) were measured using a NanoDrop 2000. Each microarray slide (catalog no. p/n G2534-60011/G2534-60014; Agilent Technologies Inc.) was hybridized with $1.65 \mu \mathrm{g}$ Cy3-labeled cRNA using a gene expression hybridization kit (catalog no. $\mathrm{p} / \mathrm{n}$ 5188-5242; Agilent Technologies, Inc.) in a hybridization oven (catalog no. p/n G2545A; Agilent Technologies, Inc.), according to the manufacturer's protocol. Following $17 \mathrm{~h}$ of hybridization, the slides were washed in staining dishes (Thermo Fisher Scientific, Inc.) with a gene expression wash buffer kit (catalog no. p/n 5188-5327; Agilent Technologies, Inc.), following the manufacturer's protocol. Next, the slides were scanned using an Agilent Microarray Scanner G2565C (Agilent Technologies, Inc.) with the following settings: Dye channel green, scan resolution $3 \mu \mathrm{m}$, PMT $100 \%$ and 20-bit scanning. The Agilent Feature Extraction software (version 10.7; Agilent Technologies, Inc.) was used to analyze the acquired array images. Quantile normalization and subsequent data processing were performed using GeneSpring software version 11.0 (Agilent Technologies, Inc.). DEGs were identified through fold change ( $>2$-fold) filtering. Microarray analysis was performed by Shanghai Biotechnology Corporation (Shanghai, China). Array data were deposited at the Gene Expression Omnibus database of the National Center for Biotechnology Information (accession no. GSE81922).

Functional enrichment analysis. To further understand the biological relevance and associated pathways of DEGs, functional enrichment analysis was performed using the Biological Network Gene Ontology (BiNGO; v3.0.3) and CluePedia (v1.0.4) web-based tools (27,28). BiNGO (www.psb.ugent. be/cbd/papers/BiNGO) is a tool that identifies Gene Ontology (GO) terms that are significantly overrepresented in a set of genes or a subgraph of a biological network. BiNGO maps the predominant functional themes of the tested gene set on the GO hierarchy and takes advantage of Cytoscape's versatile visualization environment to produce an intuitive molecular interaction network. The CluePediaCytoscape plugin (v3.0.1; www.ici.upmc.fr/cluepedia) is a search tool for new markers that are potentially associated to pathways. A pathway-like visualization can be created using the Cerebral plugin (v2.8.2) layout (29). The threshold of hypergeometric distribution of functional annotation was 0.05 .

Construction of interaction networks. Since genes act by interacting with other genes to accomplish their functions; the interaction networks of the candidate genes identified were further explored by bioinformatics analysis. In the 
Table I. Differentially-expressed genes in M1 vs. M2 polarized macrophages.

\begin{tabular}{|c|c|c|c|c|c|}
\hline Probe name & Gene symbol & P-value & Fold change & $\mathrm{FC}(\mathrm{abs})$ & Regulation \\
\hline A_51_P257951 & Retnla & 0.0041927 & 0.00014303 & 6991.6038 & Down \\
\hline A_51_P167292 & CHI3L3 & $6.022 \mathrm{E}-05$ & 0.00244865 & 408.38827 & Down \\
\hline A_55_P1988108 & MRC1 & 0.0144366 & 0.01116567 & 89.560221 & Down \\
\hline A_55_P2158741 & NOS2 & 0.0267168 & 80.8592825 & 80.859282 & Up \\
\hline A_66_P116173 & IL23r & 0.00021806 & 60.0522186 & 60.0522186 & Up \\
\hline A_51_P303160 & ARG1 & 0.0001499 & 0.02261723 & 44.214073 & Down \\
\hline A_51_P106799 & PPARG & 0.00702976 & 0.048704658 & 20.531917 & Down \\
\hline A_51_P107362 & SOCS2 & 0.0016812 & 0.048945465 & 20.4309019 & Down \\
\hline A_55_P1992834 & SOCS2 & 0.00505959 & 0.056061637 & 17.8375098 & Down \\
\hline A_51_P322640 & CCL24 & 0.02594911 & 0.067245489 & 14.870886 & Down \\
\hline A_55_P1992838 & SOCS2 & 0.00031572 & 0.072890051 & 13.7192935 & Down \\
\hline A_51_P474459 & SOCS3 & 0.00465443 & 9.357196051 & 9.35719605 & Up \\
\hline A_51_P212782 & IL1b & 0.01326346 & 7.485790577 & 7.48579058 & Up \\
\hline A_55_P1997756 & IL6 & 0.00478943 & 7.184303002 & 7.184303 & Up \\
\hline A_51_P385099 & $\mathrm{TNF}$ & 0.0009646 & 6.838318605 & 6.8383186 & Up \\
\hline A_51_P473888 & IL6st & 0.003416 & 0.162871741 & 6.13980053 & Down \\
\hline A_55_P2082974 & IRAK2 & 0.02073071 & 2.412076065 & 2.41207607 & Up \\
\hline A_52_P356204 & NOSTRIN & 0.00827602 & 0.419123778 & 2.38593001 & Down \\
\hline A_51_P271503 & IL1r1 & 0.00793288 & 0.450111469 & 2.22167189 & Down \\
\hline A_51_P387608 & HIF1a & 0.01494099 & 2.111818487 & 2.11181849 & Up \\
\hline
\end{tabular}

FC (abs), fold change absolute; Retnla, resistin-like $\alpha$; CHI3L3, chitinase 3-like 3; MRC1, mannose receptor C-type 1; NOS2, nitric oxide synthase 2; IL, interleukin; ARG1, arginase 1; PPARG, peroxisome proliferator-activated receptor; SOCS, suppressor of cytokine signaling; CCL24, C-C motif chemokine ligand 24; TNF, tumor necrosis factor; IRAK2, interleukin 1 receptor associated kinase 2; NOSTRIN, nitric oxide synthase trafficker; HIF1a, hypoxia-inducible factor $1 \alpha$.

present study, 18 macrophage polarization-associated genes identified by gene expression profiling (listed in Table I) were examined for gene interaction networks using the Search for the Retrieval of Interacting Genes/Proteins (STRING; v9.0) database (string-db.org) (30). This database provides information on both experimental and predicted interactions from varied sources, including computational prediction, literature mining and knowledge transfer between organisms and information aggregated from other primary databases. An extended network was constructed by setting the required confidence score to 0.400 .

Statistical analysis. The threshold set for significant up- and downregulated DEGs in microarray data was $>2$-fold change and $\mathrm{P}<0.05$. Data were expressed as the mean \pm standard error of the mean. Statistical analysis was performed using a Student's t-test by using Graphpad Prism v5.0 (GraphPad Software, Inc., La Jolla, CA, USA) for comparison between two groups. $\mathrm{P}<0.05$ was considered to indicate a statistically significant difference.

\section{Results}

Overview of DEG profiles in M1 and M2 macrophages. A box-plot was used to visualize the distributions of the intensities from all samples, and principal component analysis (PCA) was employed to perform an unsupervised examination of differences in the signals between M1 macrophages and M2 macrophages. As demonstrated in Fig. 1A, the distribution of the $\log 2$-ratio of the microarray intensity values in the six samples (three repeats for M1 and three repeats for M2 macrophages) was very similar following quantile normalization. The M1 macrophage samples were distinctly separated from the M2 macrophage samples in the PCA plots (Fig. 1B), suggesting a differential gene expression between M1 and M2 macrophages.

Based on a threshold set at $>2$-fold change and $\mathrm{P}<0.05$ for the microarray data, a total of 1,253 differentially-expressed mRNAs were identified in M1 compared with M2 macrophage samples, of which 696 mRNAs were upregulated and 557 mRNAs were downregulated. A volcano plot illustrated the expression variance in the number of DEGs at different P-values and fold changes (Fig. 1C). Independent hierarchical clustering, visualized by a heat map (Fig. 1D), further confirmed that the identified DEGs were significantly distinct between the M1 and M2 groups.

GO and pathway analyses of DEGs. To generate insights into the potential biological functions of DEGs, functional enrichment analysis was performed using GO and KEGG pathway terms and mapped in functional networks using the Cytoscape plug-ins, BiNGO and CluePedia. GO identified three categories: biological process, cellular component, and molecular function. Through GO analysis, 34 and $40 \mathrm{GO}$ 
A

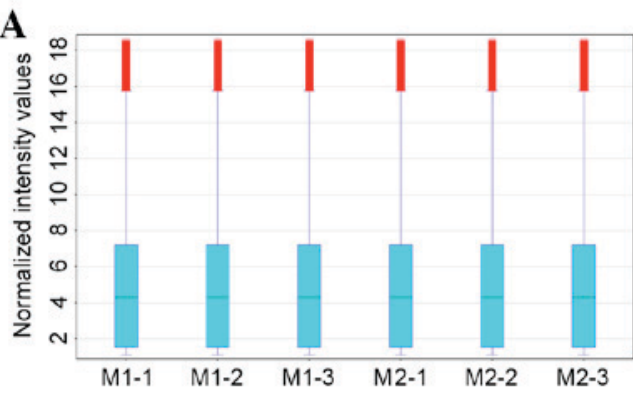

C

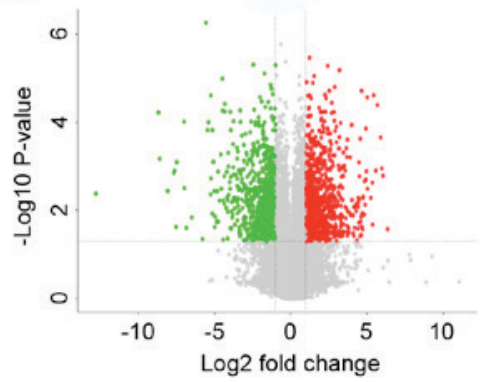

B

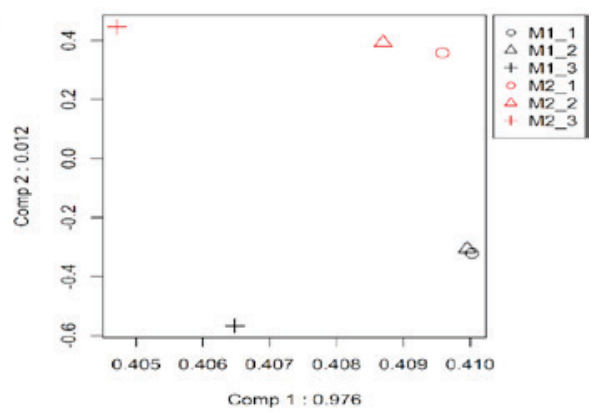

D

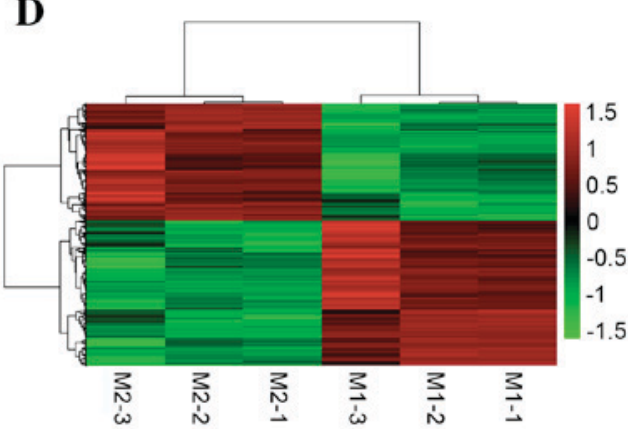

Figure 1. Validation of microarray data. (A) Box plot visualization of distribution of intensities for all samples analyzed by microarray. (B) Principal component analysis for the M1 and M2 macrophage groups based on the 1,253 differentially-expressed genes. Black illustrates the M1 macrophage samples, and red represents the M2 macrophage samples. (C) Volcano plot comparing the levels of gene expression between M1 macrophages and M2 macrophages. Red and green dots represent upregulated and downregulated mRNAs ( $>2.0$-fold change and $\mathrm{P}<0.05)$, respectively. (D) Heat map of mRNA expression profiles discriminating M1 macrophage from M2 macrophage samples. Each column represents the indicated sample; each row indicates a significant fold-change in mRNA. Upregulated and downregulated genes are indicated in red and green, respectively. n=3 for each group. M1, M1 polarized macrophages; M2, M2 polarized macrophages.

A

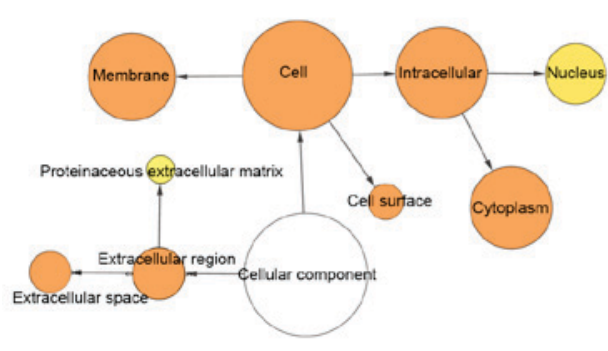

D

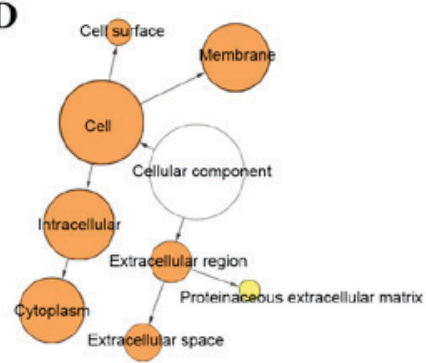

B

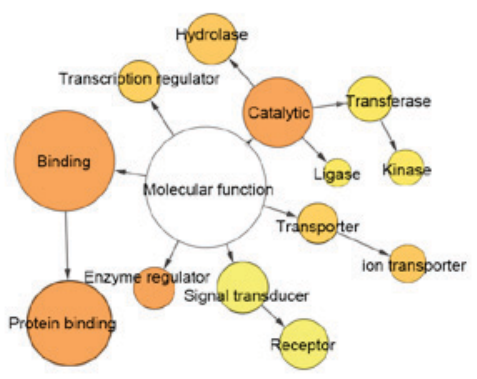

C
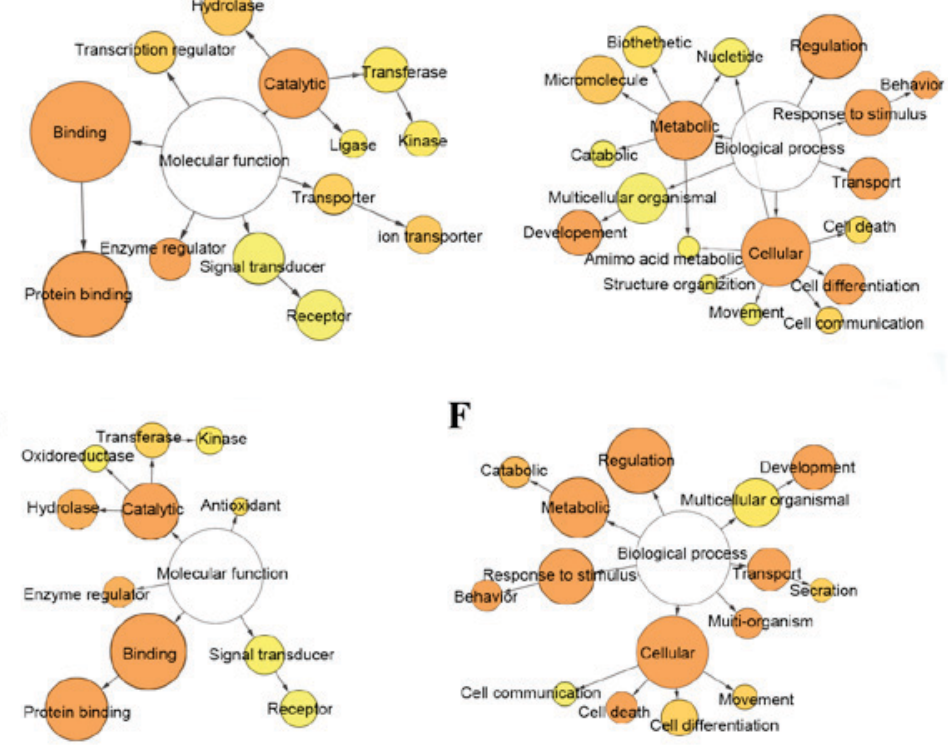

F

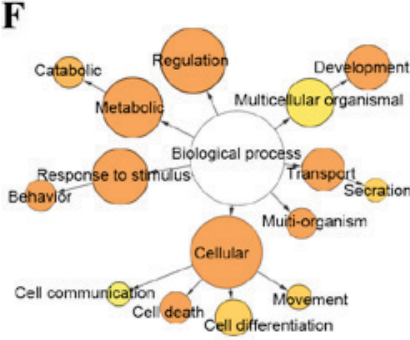

Figure 2. Differentially-expressed gene GO-term networks generated using BiNGO. Illustration of downregulated gene GO enrichment categories (A) CC, (B) MF and (C) BP. Illustration of upregulated gene GO enrichment categories (D) CC, (E) MF and (F) BP. Circle size represents GO hierarchy; the larger area of the circle, the higher hierarchy of the GO-term. Yellow shades represent enrichment level; the deeper the shade, the more significant the enrichment level. The threshold of hypergeometric distribution of the functional annotation was set at $\mathrm{P}<0.05$ and FDR $<0.05$. GO, gene ontology; BiNGO, Biological Network Gene Ontology; FDR, false discovery rate; CC, cellular component; MF, molecular function; BP, biological process.

terms were significantly enriched for up- and downregulated DEGs, respectively, based on the setting threshold of $\mathrm{P}<0.05$ and false discovery rate (FDR) $<0.05$ (Table II). The main
GO categories were: Protein binding, regulation of biological process, response to stimulus, metabolic process and cell differentiation (Fig. 2). Moreover, 15 and four pathways 
Table II. Functional annotation of differentially-expressed genes via GO enrichment.

\begin{tabular}{|c|c|c|c|}
\hline GO identifier & Description & Corrected P-value & Gene coun \\
\hline \multicolumn{4}{|c|}{ Upregulated genes } \\
\hline 50896 & Response to stimulus & $3.55 \mathrm{E}-35$ & 133 \\
\hline 5623 & Cell & $3.29 \mathrm{E}-29$ & 345 \\
\hline 5488 & Binding & $6.81 \mathrm{E}-27$ & 277 \\
\hline 5515 & Protein binding & $1.23 \mathrm{E}-24$ & 180 \\
\hline 9987 & Cellular process & $2.79 \mathrm{E}-22$ & 242 \\
\hline 16020 & Membrane & $1.11 \mathrm{E}-20$ & 210 \\
\hline 50789 & Regulation of biological process & $5.12 \mathrm{E}-20$ & 195 \\
\hline 5615 & Extracellular space & $9.41 \mathrm{E}-19$ & 46 \\
\hline 5737 & Cytoplasm & $1.07 \mathrm{E}-17$ & 190 \\
\hline 5622 & Intracellular & $6.92 \mathrm{E}-14$ & 233 \\
\hline 3824 & Catalytic activity & $1.02 \mathrm{E}-12$ & 139 \\
\hline 51704 & Multi-organism process & $2.10 \mathrm{E}-12$ & 30 \\
\hline 5576 & Extracellular region & $1.27 \mathrm{E}-11$ & 66 \\
\hline 8219 & Cell death & $1.42 \mathrm{E}-09$ & 31 \\
\hline 8152 & Metabolic process & $1.69 \mathrm{E}-09$ & 159 \\
\hline 7610 & Behavior & 7.73E-09 & 28 \\
\hline 7275 & Multicellular organismal development & $8.00 \mathrm{E}-08$ & 79 \\
\hline 6810 & Transport & $1.65 \mathrm{E}-07$ & 71 \\
\hline 9986 & Cell surface & $5.58 \mathrm{E}-07$ & 20 \\
\hline 30234 & Enzyme regulator activity & $1.98 \mathrm{E}-06$ & 29 \\
\hline 16787 & Hydrolase activity & $2.57 \mathrm{E}-06$ & 62 \\
\hline 9056 & Catabolic process & 8.94E-06 & 32 \\
\hline 6928 & Cellular component movement & $1.30 \mathrm{E}-04$ & 18 \\
\hline 30154 & Cell differentiation & $1.47 \mathrm{E}-04$ & 48 \\
\hline 46903 & Secretion & $1.49 \mathrm{E}-04$ & 14 \\
\hline 16740 & Transferase activity & $1.96 \mathrm{E}-04$ & 46 \\
\hline 16209 & Antioxidant activity & $6.51 \mathrm{E}-04$ & 5 \\
\hline 32501 & Multicellular organismal process & $2.61 \mathrm{E}-03$ & 96 \\
\hline 16301 & Kinase activity & $2.78 \mathrm{E}-03$ & 24 \\
\hline 16491 & Oxidoreductase activity & 4.81E-03 & 21 \\
\hline 4871 & Signal transducer activity & 8.77E-03 & 61 \\
\hline 5578 & Proteinaceous extracellular matrix & 2.39E-02 & 10 \\
\hline 4872 & Receptor activity & $3.77 \mathrm{E}-02$ & 53 \\
\hline 7154 & Cell communication & $4.28 \mathrm{E}-02$ & 15 \\
\hline \multicolumn{4}{|c|}{ Downregulated genes } \\
\hline 5623 & Cell & $3.0026 \mathrm{E}-32$ & 328 \\
\hline 5488 & Binding & $6.1503 \mathrm{E}-31$ & 268 \\
\hline 5515 & Protein binding & 7.6309E-31 & 182 \\
\hline 50789 & Regulation of biological process & $9.9221 \mathrm{E}-20$ & 183 \\
\hline 16020 & Membrane & $2.2576 \mathrm{E}-19$ & 194 \\
\hline 9987 & Cellular process & $3.2737 \mathrm{E}-19$ & 219 \\
\hline 5737 & Cytoplasm & $7.2487 \mathrm{E}-15$ & 171 \\
\hline 50896 & Response to stimulus & $1.2201 \mathrm{E}-13$ & 87 \\
\hline 5622 & Intracellular & $2.213 \mathrm{E}-13$ & 216 \\
\hline 7275 & Multicellular organismal development & $2.9385 \mathrm{E}-11$ & 84 \\
\hline 8152 & Metabolic process & $2.0536 \mathrm{E}-10$ & 152 \\
\hline 30154 & Cell differentiation & $2.1508 \mathrm{E}-10$ & 61 \\
\hline 5576 & Extracellular region & 4.247E-09 & 57 \\
\hline 30234 & Enzyme regulator activity & $1.0124 \mathrm{E}-08$ & 32 \\
\hline 5615 & Extracellular space & 2.4893E-08 & 29 \\
\hline 3824 & Catalytic activity & 6.7488E-08 & 115 \\
\hline
\end{tabular}


Table II. Continued.

\begin{tabular}{|c|c|c|c|}
\hline GO identifier & Description & Corrected P-value & Gene count \\
\hline 6810 & Transport & $1.433 \mathrm{E}-07$ & 67 \\
\hline 9986 & Cell surface & $1.4591 \mathrm{E}-07$ & 20 \\
\hline 32501 & Multicellular organismal process & 1.8029E-07 & 108 \\
\hline 7610 & Behavior & 4.5276E-06 & 22 \\
\hline 43170 & Macromolecule metabolic process & 0.00004114 & 96 \\
\hline 15075 & Ion transmembrane transporter activity & 4.7081E-05 & 24 \\
\hline 16787 & Hydrolase activity & 4.9943E-05 & 54 \\
\hline 7154 & Cell communication & 0.00010978 & 21 \\
\hline 30528 & Transcription regulator activity & 0.00013308 & 33 \\
\hline 5215 & Transporter activity & 0.00026927 & 30 \\
\hline 8219 & Cell death & 0.00052849 & 19 \\
\hline 9058 & Biosynthetic process & 0.00075689 & 62 \\
\hline 5634 & Nucleus & 0.0020871 & 83 \\
\hline 16740 & Transferase activity & 0.0025576 & 39 \\
\hline 6519 & Cellular amino acid and derivative metabolic process & 0.00291 & 12 \\
\hline 16301 & Kinase activity & 0.0081283 & 21 \\
\hline 9056 & Catabolic process & 0.0081283 & 22 \\
\hline 6139 & Nucleobase & 0.010583 & 55 \\
\hline 5578 & Proteinaceous extracellular matrix & 0.012461 & 10 \\
\hline 43062 & Extracellular structure organization & 0.013556 & 7 \\
\hline 4871 & Signal transducer activity & 0.01531 & 55 \\
\hline 6928 & Cellular component movement & 0.017115 & 12 \\
\hline 4872 & Receptor activity & 0.034186 & 49 \\
\hline 16874 & Ligase activity & 0.046244 & 10 \\
\hline
\end{tabular}

GO, Gene Ontology.

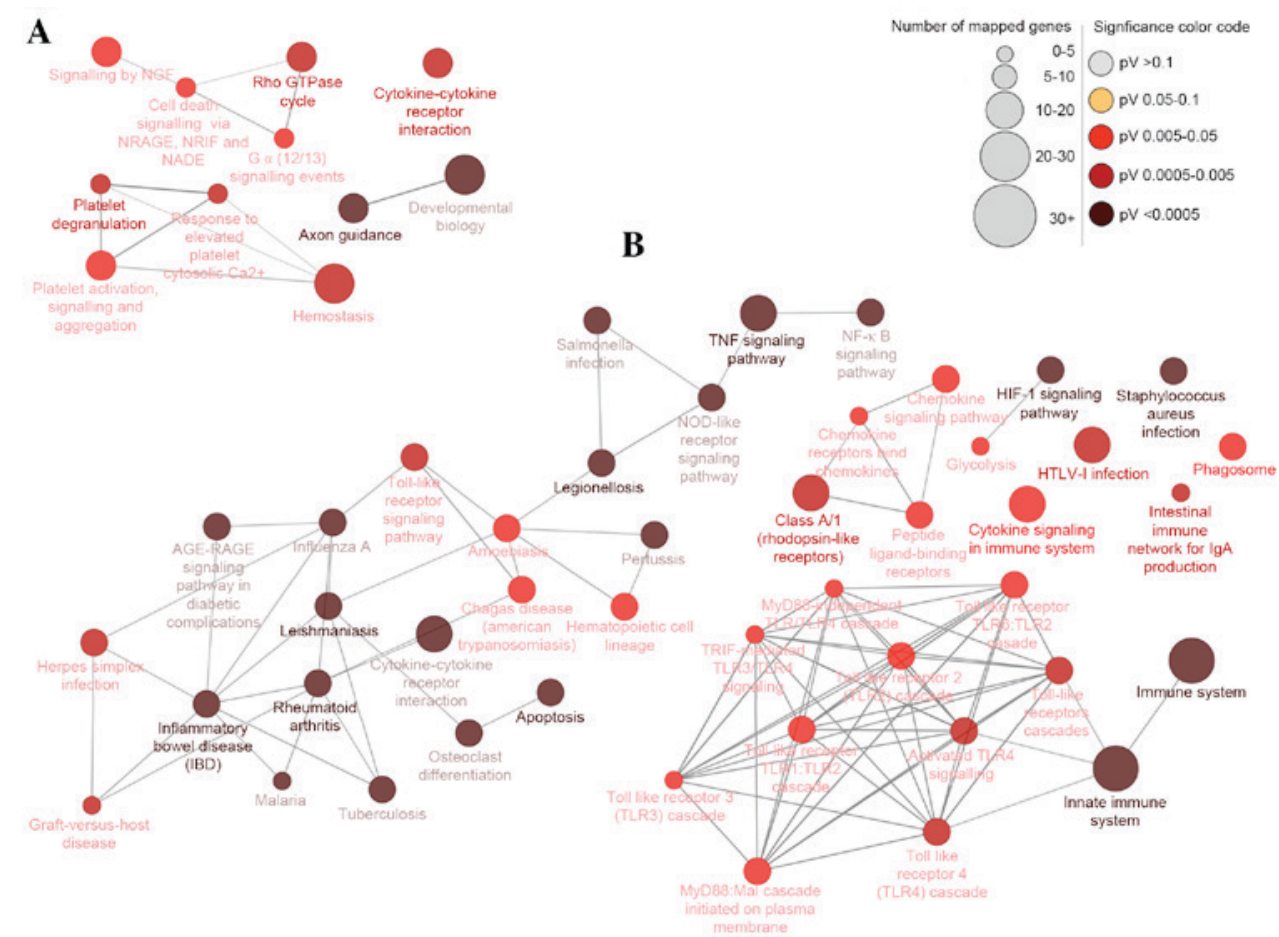

Figure 3. Differentially-expressed gene pathway network generated using CluePedia. Interaction pathway networks for the identified (A) downregulated and (B) upregulated genes. The size of the circle indicates the number of genes involved in the pathway, and the color of the circle represents the P-value. The threshold for the analysis was set at $\mathrm{P}<0.05$ and FDR $<0.05$. FDR, false discovery rate; NGF, nerve growth factor; NRAGE, MAGE family member D1; NRIF, neurotrophin receptor interacting factor; NADE, NAD synthetase; TNF, tumor necrosis factor; NFkB, nuclear factor $\kappa \mathrm{B}$; NOD, atrophin 1; RAGE, receptor for advanced glycation end products; HIF1, hypoxia-inducible factor 1; HTLV-I, human T-lymphotropic virus I; MyD88, myeloid differentiation primary response gene 88; TRIF, toll-like receptor adaptor molecule 2. 
Table III. Functional annotation of differentially-expressed genes via KEGG Enrichment.

\begin{tabular}{|c|c|c|}
\hline Function & Groups & $\begin{array}{l}\text { Gene } \\
\text { count }\end{array}$ \\
\hline \multicolumn{3}{|l|}{ Upregulated genes } \\
\hline Apoptosis & Group 9 & 29 \\
\hline Class A/1 (Rhodopsin-like receptors) & Group 8 & 30 \\
\hline Cytokine Signaling in immune system & None 4 & 21 \\
\hline HIF1 signaling pathway & Group 5 & 17 \\
\hline HTLV-I infection & None 3 & 22 \\
\hline Immune system & Group 6 & 62 \\
\hline Inflammatory bowel disease (IBD) & Group 4 & 67 \\
\hline Innate immune system & Group 7 & 36 \\
\hline $\begin{array}{l}\text { Intestinal immune network for } \\
\text { IgA production }\end{array}$ & None 1 & 8 \\
\hline Legionellosis & Group 3 & 41 \\
\hline Leishmaniasis & Group 1 & 42 \\
\hline Phagosome & None 0 & 15 \\
\hline Rheumatoid arthritis & Group 2 & 32 \\
\hline Staphylococcus aureus infection & None 2 & 12 \\
\hline TNF signaling pathway & Group 0 & 43 \\
\hline \multicolumn{3}{|l|}{ Downregulated genes } \\
\hline Axon guidance & Group 1 & 24 \\
\hline Cytokine-cytokine receptor interaction & None 0 & 18 \\
\hline Platelet degranulation & Group 0 & 24 \\
\hline Rho GTPase cycle & Group 2 & 22 \\
\hline
\end{tabular}

KEGG, Kyoto Encyclopedia of Genes and Genomes; HIF1, hypoxia-inducible factor 1; HTLV-I, human T-lymphotropic virus I; TNF, tumor necrosis factor.

were significantly enriched for up and downregulated DEGs, respectively, which could be categorized into 15 and four groups, respectively. The groups were classified according to their different functions and the function details are presented in Table III (left column). Some of the groups shared similar genes. The main pathways identified by KEGG were the HIF1 signaling pathway, TNF signaling pathway, innate immune system, apoptosis and cytokine-cytokine receptor interaction (Fig. 3).

Interaction network analysis. An interaction network was constructed using STRING and then visualized using Cytoscape based on the macrophage polarization-associated genes identified in the present study. The network comprised 18 genes and 38 interactions (Fig. 4). The main type of gene associations was co-occurrence. Among these, IL6, TNF, IL1 $\beta$, nitric oxide synthase 2 (NOS2) and SOCS3 were the key nodes, displaying the highest connectivity within the network (Fig. 4).

\section{Discussion}

Macrophages, as major innate immune and antigen presenting cells, are important in infection resistance and tumorigenesis.

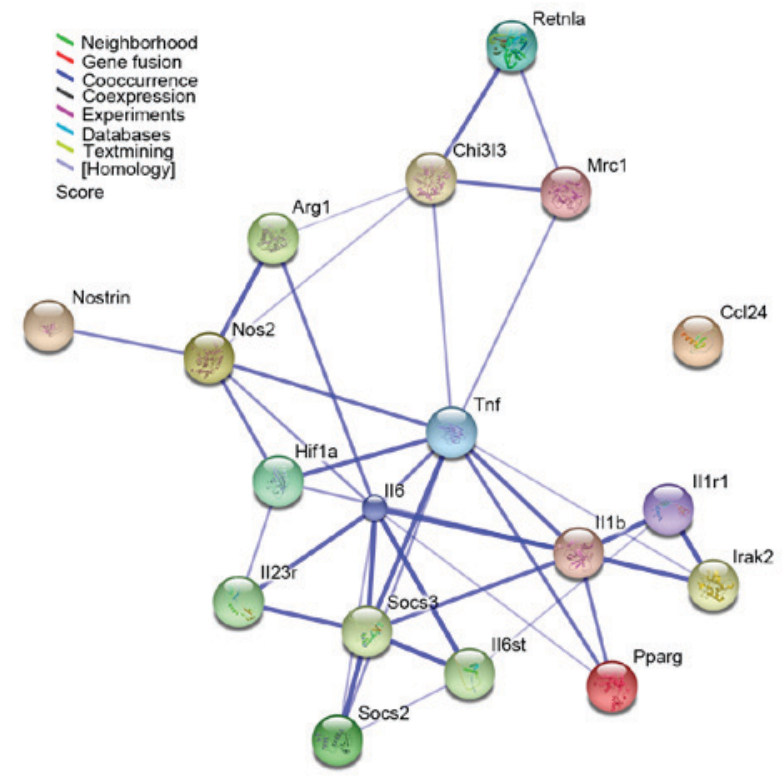

Figure 4. Interaction network of 18 macrophage polarization-associated genes as identified by STRING analysis. The results were expanded to the current network by setting the required confidence score to 0.400 . The nodes represent the genes, whereas the lines represent interactions between genes. The color of the line denotes the basis of the predicted interaction according to the software database. STRING, Search Tool for the Retrieval of Interacting Genes; Retnla, resistin-like $\alpha$; Chi313, chitinase 3-like 3; Mrc1, mannose receptor C-type 1; Arg1, arginase 1; Nostrin, nitric oxide synthase trafficker; Nos2, nitric oxide synthase 2; Hif1, hypoxia-inducible factor 1; Tnf, tumor necrosis factor; Ccl24, C-C motif chemokine ligand 24; Il, interleukin; Socs, suppressor of cytokine signaling; Irak2, interleukin 1 receptor associated kinase 2; Pparg, peroxisome proliferator-activated receptor.

Macrophages activated by TLR ligands, such as LPS or IFN- $\gamma$, are called M1 macrophages. In contrast, stimulation of macrophages with T helper cells type 2 cytokines, such as IL-4 or IL-13, induces the generation of M2-type macrophages. Treatment of bone marrow cells with granulocyte-macrophage colony-stimulating factor (CSF) and macrophage CSF, leads to the generation of M1 and M2 macrophages, respectively (31). Appropriately activated macrophages eliminate pathogens and tumors, whereas, activation with inappropriate stimuli may suppress the immune system, resulting in tumorigenesis and chronic infections. As the primary cells that secrete inflammatory cytokines, macrophages (particularly M2-type) directly mediate the development of inflammatory autoimmune diseases, tissue damage and inflammatory infiltration in hypersensitivity reactions (32-35).

Macrophage polarization has been a topic of intense interest in macrophage research. Early studies identified a number of genes involved in macrophage polarization. For example, previous studies have demonstrated that the JMJD3-interferon regulatory factor (Irf) 4 axis regulates M2 macrophage polarization and host responses against helminth infections (21). SOCS2 and SOCS3 diametrically control macrophage polarization (22). Formyl peptide receptor (FPR) 2 promotes antitumor host defense by limiting M2 polarization of macrophages (36). IRF5 and IRF8 promote M1 macrophage polarization $(14,15)$, while KLF4 is involved in M2 macrophage polarization (16). Akt1 and Akt2 protein kinases differentially contribute to macrophage polarization (17). However, although several genes associated with 
macrophage polarization have been identified, the interaction among genes and the mechanism of this constellation of genes in the response of macrophages to polarizing conditions remain elusive.

The accessibility of microarray data and gene profiling has facilitated a better understanding of the underlying mechanisms of complex biological processes and responses. In the present study, mRNA-based microarray methods were employed to analyze RNA samples from ex vivo programmed M1 and M2 macrophages isolated from BALB/c mice. Bioinformatics analysis identified a total of 1,253 DEGs in M1 macrophages, including 696 upregulated genes and 557 downregulated genes relative to M2 macrophages. Previous studies have examined the gene expression profiles of M1 and M2 macrophages derived from C57BL/6J mice and from human blood samples $(37,38)$. In the present microarray study, all 8 genes corresponding to canonical M1 markers (NOS2, IL23 receptor, SOCS3, IL-1 $\beta$, IL-6, TNF, interleukin 1 receptor associated kinase 2 and HIF1a) and the M1 markers CD38, G-protein coupled receptor (Gpr)18 and Fpr2, identified in C57BL/6 murine macrophages (37), were demonstrated to be upregulated in M1 compared with M2 macrophages (Table I). In addition, 10 genes corresponding to canonical M2 markers (including Retnla, Chi313, MRC1, ARG1 andPPARG), and the M2 markers early growth response 2 and c-myc identified in C57BL/6 murine macrophages (37), were demonstrated to be up-regulated in M2 compared with M1 macrophages in the present study (Table I). These data validate the robustness of the microarray results presented in the current study.

A better understanding of the gene functions and molecular pathways associated with different macrophage subtypes is necessary for further progress in the macrophage field. In the present study, a gene expression analysis of M1 and M2 macrophages derived from BALB/c mice was performed. The bioinformatics analysis demonstrated that, for the upregulated genes, GO functional analysis identified 34 enriched terms, including eight cellular components, 11 molecular functions and 15 biological process terms. Biological process terms comprised of response to stimulus, cell differentiation and regulation of biological process. KEGG functional analysis identified 15 enriched terms, which included apoptosis, cytokine signaling in immune system, HIF1 signaling pathway, innate immune system, and TNF signaling pathway. For the downregulated genes, GO functional analysis identified 40 enriched terms, which consisted of nine cellular components, 13 molecular functions and 18 biological process terms. KEGG functional analysis identified four enriched terms, namely, axon guidance, cytokine-cytokine receptor interaction, platelet degranulation and Rho GTPase cycle. Interaction network analysis of the screened DEGs, generated by STRING, indicated that genes including TNF, IL-6, IL-1 $\beta$, SOCS3, NOS2 and HIFla may serve key roles in macrophage polarization.

In summary, the current study identified 1,253 DEGs and analyzed their functions through GO and KEGG pathway enrichment analyses. Subsequently, an interaction network was constructed to analyze the overlapping DEGs with known genes associated with macrophage polarization. The present study may thus provide novel insights into the role of genes in macrophage differentiation and polarization. Further experimental studies will be needed in the future in order to confirm these findings and further explore the molecular mechanisms of macrophage polarization.

\section{Acknowledgements}

The National Natural Science Foundation of China (grant nos. 81300172, 81301497 and 81472017), Natural Science Foundation of Anhui Province (grant no. 1408085QH148), Key projects of Natural Science Research of universities in Anhui Province (grant no. KJ2016A721) and Program for Excellent Young Talents in College and University of Anhui Province supported the present study.

\section{References}

1. Liu G and Abraham E: MicroRNAs in immune response and macrophage polarization. Arterioscler Thromb Vasc Biol 33: 170-177, 2013.

2. Tugal D, Liao X and Jain MK: Transcription control of macrophage polarization. Arterioscler Thromb Vasc Biol 33: 1135-1144, 2013.

3. Stefater JA III, Ren S, Lang RA and Duffield JS: Metchnikoff's policemen: Macrophages in development, homeostasis and regeneration. Trends Mol Med 17: 743-752, 2011.

4. Lawrence $T$ and Natoli G: Transcriptional regulation of macrophage polarization: Enabling diversity with identity. Nat Rev Immunol 11: 750-761, 2011.

5. Murray PJ and Wynn TA: Obstacles and opportunities for understanding macrophage polarization. J Leukoc Biol 89: 557-563, 2011.

6. Zhang Y, Zhang M, Zhong M, Suo Q and Lv K: Expression profiles of miRNAs in polarized macrophages. Int J Mol Med 31: 797-802, 2013.

7. Mosser DM: The many faces of macrophage activation. J Leukoc Biol 73: 209-212, 2003.

8. Murray PJ, Allen JE, Biswas SK, Fisher EA, Gilroy DW, Goerdt S, Gordon S, Hamilton JA, Ivashkiv LB, Lawrence T, et al: Macrophage activation and polarization: Nomenclature and experimental guidelines. Immunity 41: 14-20, 2014.

9. Bronte $\mathrm{V}$ and Zanovello P: Regulation of immune responses by L-arginine metabolism. Nat Rev Immunol 5: 641-654, 2005.

10. Nair MG, Gallagher IJ, Taylor MD, Loke P, Coulson PS, Wilson RA, Maizels RM and Allen JE: Chitinase and Fizz family members are a generalized feature of nematode infection with selective upregulation of Ym1 and Fizz1 by antigen-presenting cells. Infect Immun 73: 385-394, 2005.

11. Stein M, Keshav S, Harris N and Gordon S: Interleukin 4 potently enhances murine macrophage mannose receptor activity: A marker of alternative immunologic macrophage activation. J Exp Med 176: 287-292, 1992.

12. Mantovani A, Sica A, Sozzani S, Allavena P, Vecchi A and Locati M: The chemokine system in diverse forms of macrophage activation and polarization. Trends Immunol 25: 677-686, 2004.

13. Wang N, Liang H and Zen K: Molecular mechanisms that influence the macrophage m1-m2 polarization balance. Front Immunol 5: 614, 2014.

14. Krausgruber T, Blazek K, Smallie T, Alzabin S, Lockstone H, Sahgal N, Hussell T, Feldmann M and Udalova IA: IRF5 promotes inflammatory macrophage polarization and TH1-TH17 responses. Nat Immunol 12: 231-238, 2011.

15. Xu H, Zhu J, Smith S, Foldi J, Zhao B, Chung AY, Outtz H, Kitajewski J, Shi C, Weber S, et al: Notch-RBP-J signaling regulates the transcription factor IRF8 to promote inflammatory macrophage polarization. Nat Immunol 13: 642-650, 2012.

16. Liao X, Sharma N, Kapadia F, Zhou G, Lu Y, Hong H, Paruchuri K, Mahabeleshwar GH, Dalmas E, Venteclef N, et al: Krüppel-like factor 4 regulates macrophage polarization. J Clin Invest 121: 2736-2749, 2011.

17. Arranz A, Doxaki C, Vergadi E, Martinez de la Torre Y, Vaporidi K, Lagoudaki ED, Ieronymaki E, Androulidaki A, Venihaki M, Margioris AN, et al: Akt1 and Akt2 protein kinases differentially contribute to macrophage polarization. Proc Natl Acad Sci USA 109: 9517-9522, 2012. 
18. Zhang Y, Zhang M, Li X, Tang Z, Wang X, Zhong M, Suo Q, Zhang Y and Lv K: Silencing microRNA-155 attenuates cardiac injury and dysfunction in viral myocarditis via promotion of M2 phenotype polarization of macrophages. Sci Rep 6: 22613, 2016.

19. Takeuch $O$ and Akira S: Epigenetic control of macrophage polarization. Eur J Immunol 41: 2490-2493, 2011.

20. Mullican SE, Gaddis CA, Alenghat T, Nair MG, Giacomin PR, Everett LJ, Feng D, Steger DJ, Schug J, Artis D and Lazar MA: Histone deacetylase 3 is an epigenomic brake in macrophage alternative activation. Genes Dev 25: 2480-2488, 2011.

21. Satoh T, Takeuchi O, Vandenbon A, Yasuda K, Tanaka Y, Kumagai Y, Miyake T, Matsushita K, Okazaki T, Saitoh T, et al: The Jmjd3-Irf4 axis regulates M2 macrophage polarization and host responses against helminth infection. Nat Immunol 11: 936-944, 2010

22. Spence S, Fitzsimons A, Boyd CR, Kessler J, Fitzgerald D, Elliott J, Gabhann JN, Smith S, Sica A, Hams E, et al: Suppressors of cytokine signaling 2 and 3 diametrically control macrophage polarization. Immunity 38: 66-78, 2013.

23. Liu C, Fei HD, Sun ZY and Tian JW: Bioinformatic analysis of the microarray gene expression profile in degenerative intervertebral disc cells exposed to TNF- $\alpha$. Eur Rev Med Pharmacol Sci 19: 3332-3339, 2015

24. Zhao L, Zhang J, Tan H, Wang W, Liu Y, Song R and Wang L: Gene function analysis in osteosarcoma based on microarray gene expression profiling. Int J Clin Exp Med 8: 10401-10410, 2015.

25. Zhang Y, Zhang Y, Li X, Zhang M and Lv K: Microarray analysis of circular RNA expression patterns in polarized macrophages. Int J Mol Med 39: 373-379, 2017.

26. Boltz-Nitulescu G, Wiltschke C, Holzinger C, Fellinger A, Scheiner O, Gessl A and Förster O: Differentiation of rat bone marrow cells into macrophages under the influence of mouse L929 cell supernatant. J Leukoc Biol 41: 83-91, 1987.

27. Maere S, Heymans K and Kuiper M: BiNGO: A Cytoscape plugin to assess overrepresentation of gene ontology categories in biological networks. Bioinformatics 21: 3448-3449, 2005.

28. Bindea G, Galon J and Mlecnik B: CluePedia Cytoscape plugin: Pathway insights using integrated experimental and in silico data. Bioinformatics 29: 661-663, 2013.

29. Barsky A, Gardy JL, Hancock RE and Munzner T: Cerebral A Cytoscape plugin for layout of and interaction with biological networks using subcellular localization annotation. Bioinformatics 23: 1040-1042, 2007.
30. Szklarczyk D, Franceschini A, Kuhn M, Simonovic M, Roth A, Minguez P, Doerks T, Stark M, Muller J, Bork P, et al: The STRING database in 2011: Functional interaction networks of proteins, globally integrated and scored. Nucleic Acids Res 39 (Database Issue): D561-D568, 2011.

31. Banerjee S, Cui H, Xie N, Tan Z, Yang S, Icyuz M, Thannickal VJ, Abraham E and Liu G: miR-125a-5p regulates differential activation of macrophages and inflammation. J Biol Chem 288: 35428-35436, 2013.

32. Li K, Xu W, Guo Q, Jiang Z, Wang P, Yue Y and Xiong S: Differential macrophage polarization in male and female BALB/c mice infected with coxsackievirus B3 defines susceptibility to viral myocarditis. Circ Res 105: 353-364, 2009.

33. Chacón-Salinas R, Serafín-López J, Ramos-Payán R, Méndez-Aragón P, Hernández-Pando R, Van-Soolingen D, Flores-Romo L, Estrada-Parra $\mathrm{S}$ and Estrada-García I: Differential pattern of cytokine expression by macrophages infected in vitro with different Mycobacterium tuberculosis genotypes. Clin Exp Immunol 140: 443-449, 2005.

34. Verreck FA, de Boer T, Langenberg DM, van der Zanden $L$ and Ottenhoff TH: Phenotypic and functional profiling of human proinflammatory type- 1 and anti-inflammatory type-2 macrophages in response to microbial antigens and IFN-gamma and CD40L-mediated costimulation. J Leukoc Biol 79: 285-293, 2006.

35. Zhang W, Xu W and Xiong S: Blockade of Notch1 signaling alleviates murine lupus via blunting macrophage activation and M2b polarization. J Immunol 184: 6465-6478, 2010.

36. Liu Y, Chen K, Wang C, Gong W, Yoshimura T, Liu M and Wang JM: Cell surface receptor FPR2 promotes antitumor host defense by limiting M2 polarization of macrophages. Cancer Res 73: 550-560, 2013.

37. Jablonski KA, Amici SA, Webb LM, Ruiz-Rosado Jde D, Popovich PG, Partida-Sanchez S and Guerau-de-Arellano M: Novel markers to delineate murine M1 and M2 macrophages. PLoS One 10: e0145342, 2015.

38. Martinez FO, Gordon S, Locati $M$ and Mantovani A: Transcriptional profiling of the human monocyte-to macrophage differentiation and polarization: New molecules and patterns of gene expression. J Immunol 177: 7303-7311, 2006. 